\title{
Méditerranée
}

Numéro 115 (2010)

Rivages méditerranéens

Taieb Boumeaza, Abdelkader Sbai, Marc Salmon et André Ozer

\section{Impacts écologiques des aménagements touristiques sur le littoral de Saïdia , Maroc oriental}

\begin{abstract}
Avertissement
Le contenu de ce site relève de la législation française sur la propriété intellectuelle et est la propriété exclusive de l'éditeur.

Les œuvres figurant sur ce site peuvent être consultées et reproduites sur un support papier ou numérique sous réserve qu'elles soient strictement réservées à un usage soit personnel, soit scientifique ou pédagogique excluant toute exploitation commerciale. La reproduction devra obligatoirement mentionner l'éditeur, le nom de la revue, l'auteur et la référence du document.

Toute autre reproduction est interdite sauf accord préalable de l'éditeur, en dehors des cas prévus par la législation en vigueur en France.
\end{abstract}

\section{revues.org}

Revues.org est un portail de revues en sciences humaines et sociales développé par le Cléo, Centre pour l'édition électronique ouverte (CNRS, EHESS, UP, UAPV).

\section{Référence électronique}

Taieb Boumeaza, Abdelkader Sbai, Marc Salmon et André Ozer, «Impacts écologiques des aménagements touristiques sur le littoral de Saïdia , Maroc oriental », Méditerranée [En ligne], 115 | 2010, mis en ligne le 30 décembre 2012. URL : http://mediterranee.revues.org/index4888.html

DOI : en cours d'attribution

Éditeur : Publications de l'université de Provence

http://mediterranee.revues.org

http://www.revues.org

Document accessible en ligne sur : http://mediterranee.revues.org/index4888.html

Ce document est le fac-similé de l'édition papier.

Cet article a été téléchargé sur le portail Cairn (http://www.cairn.info).

\section{CA I R N}

Chercher. Repérer. Avancer.

Distribution électronique Cairn pour Publications de l'université de Provence et pour Revues.org (Centre pour l'édition électronique ouverte)

Tous droits réservés 


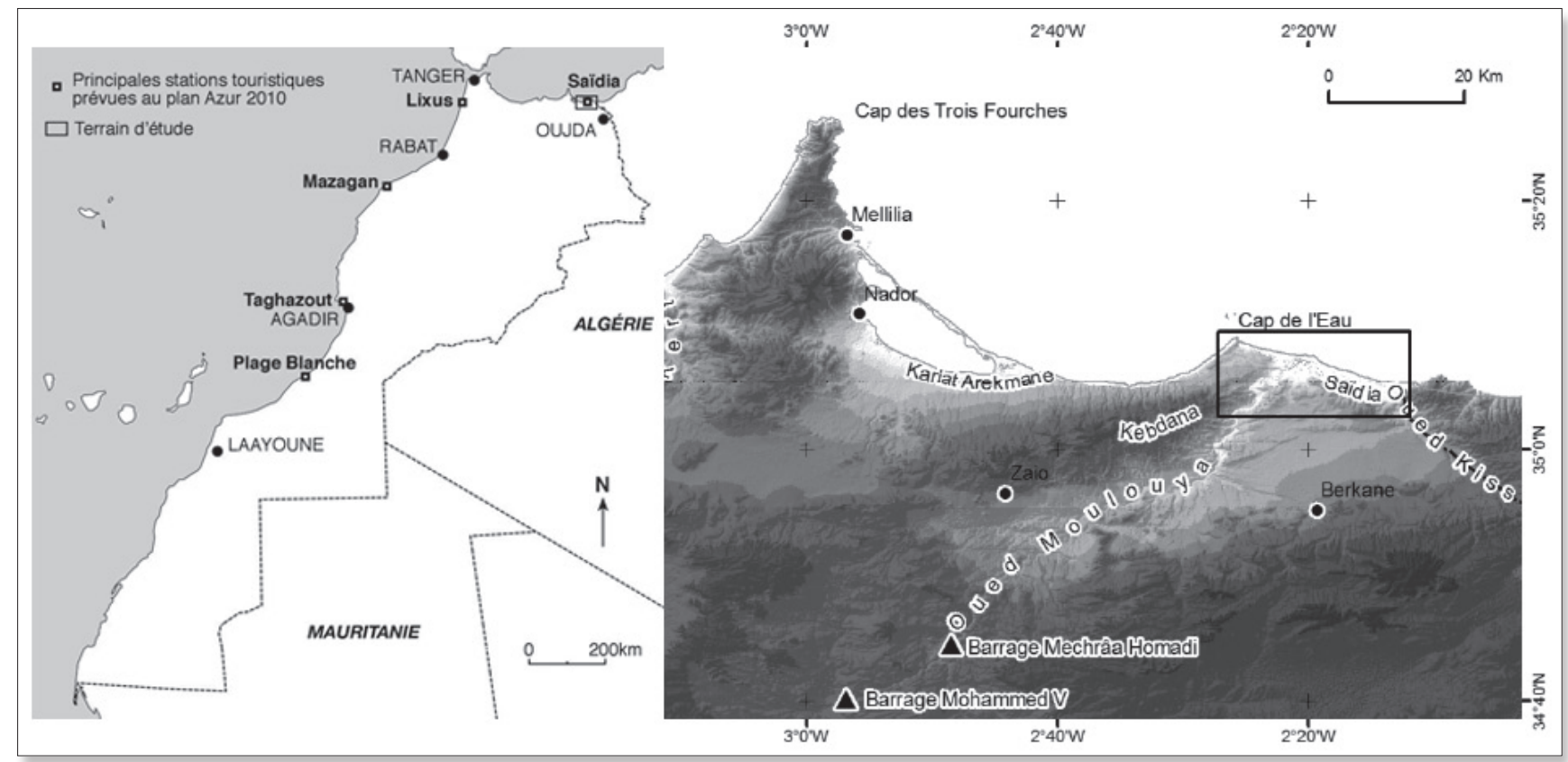

Fig. 1 - Localisation des principales stations touristiques prévues par le plan Azur et de la zone d'étude sur le littoral méditerranéen oriental Fond de carte: modèle numérique de terrain SRTM - Le SIBE est entouré d'un rectangle 


\section{Impacts écologiques des aménagements touristiques sur le littoral de Saïdia , Maroc oriental Ecological impacts of tourist developments on the coast of Saïdia, oriental Morocco}

\author{
Taieb BouMEAZA \\ Département de géographie \\ Lab. GESIA \\ Université Hassan II \\ Mohammedia-Casablanca Maroc \\ taiebboumeaza@yahoo.fr
}

\author{
Abdelkader SBAI \\ Département de géographie \\ Université Mohammed Ier \\ Oujda Maroc \\ sbai_abdelkader@yahoo.fr
}

\author{
Marc SALMON \\ Université de Liège, Sart Tilman \\ Liège Belgique \\ msalmon@ulg.ac.be
}

ANDRÉ OZER

Université de Liège, Sart Tilman

Liège Belgique aozer@ulg.ac.be
Le littoral de Saïdia est connu depuis fort longtemps pour sa plage naturelle dont l'évolution est dépendante d'une part des alluvions apportées par la Moulouya qui avait permis l'engraissement de ce littoral, créant un delta avant la construction des barrages et d'autre part des courants marins responsables de la redistribution des sables le long de la côte. Actuellement, ce littoral subit une pression anthropique aggravée, due à la construction d'une station balnéaire et d'une marina à proximité immédiate de la côte. Ceci a provoqué un déséquilibre du système dunaire et a bouleversé les mécanismes d'évolution du littoral, avec des impacts directs sur l'environnement écologique de la zone humide de la Moulouya.

Mots clés: Maroc, delta de la Moulouya, pression anthropique, risques littoraux

Le gouvernement marocain, dans le but d'imiter le modèle espagnol pour développer le tourisme de masse, a élaboré en 2001 le Plan Azur et la Vision 2010, afin d'accueillir 10 millions de touristes par an. Le Plan Azur prévoit de développer les stations touristiques suivantes le long des côtes marocaines de la Méditerranée et de l'Atlantique: Mediterrania Saïdia, Mazagan Beach Resort, Port Lixus, Mogador Essaouira, Taghazout-Argana Bay et Plage blanche-Guelmim (fig. 1). Mais aucune étude n'a tiré les leçons du désastre écologique que le tourisme de masse a provoqué sur le littoral espagnol. Ce dernier est basé en effet sur l'occupation de la première ligne de plage, au contraire du tourisme « classique » de soleil et de plage, installé dans l'immédiat arrière-pays. Les constructions d'appartements et d'hôtels de front de mer n'ont pas eu seulement un impact négatif sur l'environnement, mais les revenus de ce tourisme ont bénéficié à peu de personnes et ont encouragé la corruption politique. Mais, si le gouvernement espagnol s'est rendu compte de ses erreurs en termes de développement et d'aménagement du littoral et essaie de restaurer l'aspect naturel de ses plages, le gouvernement marocain persiste dans l'application du «Plan Azur », malgré les dégâts causés au rivage et malgré l'opposition de la société civile.

Sur le littoral méditerranéen, la construction de la station balnéaire de Saïdia (la Nouvelle Station Touristique de Saïdia (NSTS)' ) qui a été concédée au promoteur espagnol «FADESA », s'inscrit dans un environnement déjà fragilisé par les aménagements antérieurs (barrages sur la Moulouya, digue portuaire). On analysera ses impacts sur la plage de Saïdia et sur le cordon dunaire, ainsi que sur la forêt qui
The changing coastline of Saidia is due to two major factors. The first one relates to the currents that feed and distribute sand and the second relates to the contribution of the Moulouy a river, the main artery which ensures draining of this eastern part of Morocco. After the construction of dams, much of the alluvium is stopped upstream and currents have, up to now, currently prevailed with a modification of the coastline. Actually, this coastline has undergone an anthropogenic pressure due to the construction of the "Fadesa" resort by the coast. First, this has caused an imbalance in the dune system and second, the construction of a marina has immediately overwhelmed the system of coastal change. This marina has had direct impacts on the ecological environment of the Moulouya wetland.

Key-words: Maroc, Moulouya delta, anthropic pressure, coastal hazards

longeait le littoral. Cet aménagement représente une véritable menace pour le devenir du Site d'Intérêt Biologique et Écologique (SIBE) de la Moulouya.

Dans cet article, après une présentation des caractéristiques du milieu naturel, nous décrirons les aménagements réalisés dans le cadre du projet NSTS et dans son voisinage immédiat, puis nous analyserons ses impacts géomorphologiques et écologiques sur le littoral et sur le SIBE de la Moulouya.

\section{I - Méthodologie et outils de travail}

Pour réaliser cette étude, nous nous sommes basés sur le dépouillement bibliographique des recherches antérieures (ZOURARAH, 1995; BOUMEAZA, 1997, 2002, 2006 a et b; IRZI, 2002; HAGELSTEIN, 2005 ; BOUMEAZA et SAIDI, 2005; SBAI et al., 2005; SBAI, 2006,2008; MAREK, 2007 ; NAHON, 2008; SNOUSSI et al., 2008; SALMON et al., 2010), complété par un travail de terrain et l'analyse d'images.

Les relevés de terrain ont permis de suivre les dynamiques liées aux aménagements réalisés et l'état de dégradation du littoral. Ils reposent sur des mesures directes à partir de points de repères, mesurés chaque année à la même date (février) depuis 1996. Les derniers relevés ont eu lieu en 2008, après la construction du Port de plaisance de la Marina.

Les documents utilisés sont les cartes topographiques au1/50 000 de Triffa et de Saïdia (1965), qui reposent sur des données datant de 1958. Nous avons également utilisé les photographies aériennes de 1958 (1/40 000), 1963 (1/40000), 1980 (1/20000), 1988 (1/40000), $1995(1 / 20000)$ et des 
images satellitaires d'Aster (2004), GEOEYE (2009) et Google Earth (2009).

\section{2 - Caractéristiques naturelles}

\section{I - Situation géographique}

La zone d'étude est localisée à l'extrême Nord-Est du Maroc sur le littoral méditerranéen (fig. 1). Sur le plan administratif, elle fait partie de la Région de l'Oriental et des provinces de Berkane et de Nador et englobe la commune urbaine de Saïdia et les communes rurales de Madagh, Laâtamna et Ras Kebdana.

Elle est délimitée au Nord par la Méditerranée, au Sud par les collines des Oulad Mansour et de la chaîne des Kabdana, à l'Ouest par la chaîne des Kabdana et à l'Est par l'oued Kiss qui marque la frontière avec l'Algérie.

Le cadre naturel correspond à une vaste plaine côtière plane et de faible altitude (1 à $6 \mathrm{~m}$ au-dessus du niveau marin), large de $5 \mathrm{~km}$ pour $22 \mathrm{~km}$ de long (fig. 2). La présence au niveau de la plaine côtière d'une nappe phréatique salée et de sols argileux salés, rend difficile sa mise en valeur agricole. La plage de sable est donc d'un grand intérêt pour les estivants et les vacanciers à la recherche de la mer et du soleil.

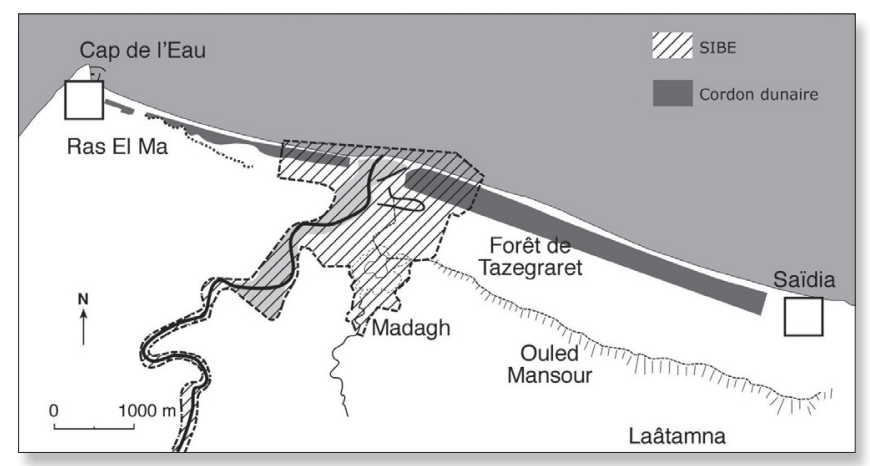

Fig. 2 - Localisation de l'embouchure et de la zone humide du SIBE de la Moulouya - Carte topographique au 1/50000, division de la cartographie, carte des Triffa

Le réseau hydrographique est constitué essentiellement par l'oued Kiss à l'est, constituant la frontière avec l'Algérie et l'oued Moulouya à l'ouest. Ils constituent les limites naturelles de la plaine de Saïdia. L'impact de l'oued Kiss est pratiquement nul pour la plaine et le littoral de Saïdia, qui ont sont issus des apports sédimentaires de la mer et de la Moulouya, avant la construction des barrages. Nos recherches ont donc porté sur le fonctionnement de cet organisme.

\section{2 - Conditions climatiques}

Le bassin de la Moulouya (superficie totale $\pm 53000 \mathrm{~km}^{2}$ ), présente une large gamme d'étages bioclimatiques (fig. 3). Dans son bassin supérieur, aux confins du haut Atlas, où l'oued prend sa source, et dans ses affluents principaux de rive gauche drainant le Moyen Atlas, le climat humide, à hiver frais et neigeux, génère des précipitations importantes et généralement régulières (600 à $900 \mathrm{~mm} / \mathrm{an}$ ). En moyenne et basse Moulouya, les conditions climatiques tendent vers un climat semi-aride à aride, où les précipitations sont sporadiques et d'importance très inégale (de 190 à $500 \mathrm{~mm} / \mathrm{an}$ ).

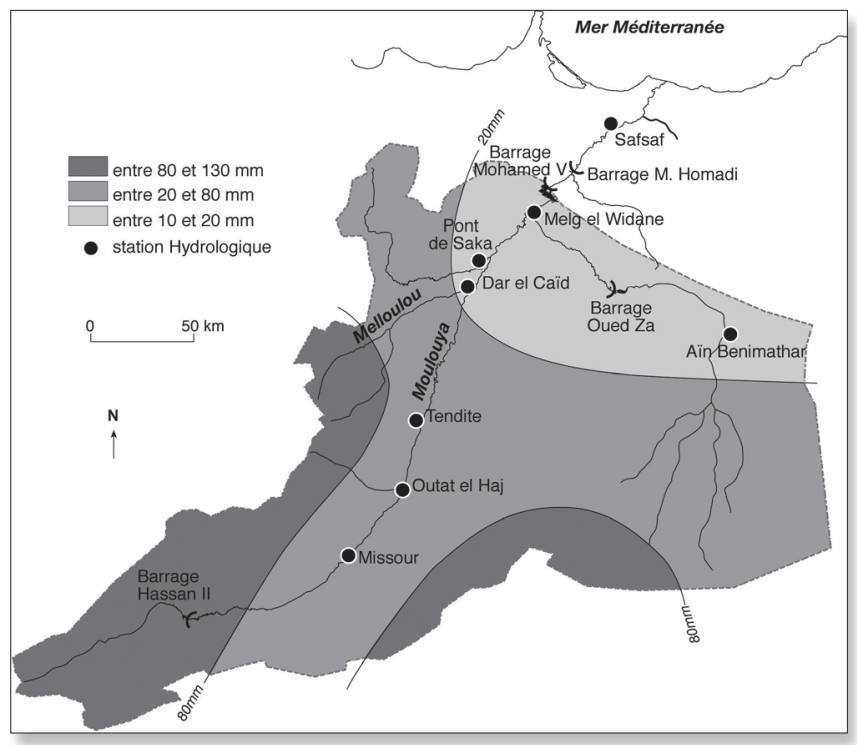

Fig. 3 - Total pluviométrique, période 12/11/93 au 15/11/93 - D'après Messouadi et Chikri (1994) modifié

La plaine littorale est caractérisée par des précipitations peu abondantes (324 mm en moyenne de 1963 à 2007) et irrégulières, avec des variations interannuelles et saisonnières importantes et une saison sèche estivale marquée. Cette variabilité explique les fluctuations des plans d'eau, en particulier celui de la nappe phréatique superficielle, qui affleure lorsque les précipitations dépassent la moyenne saisonnière. Le substrat est constitué de dépôts fins imperméables (limons fins et argiles) déposés par la Moulouya, qui sont rapidement engorgés, d'autant qu'ils reposent sur des marnes néogènes. La topographie en creux de la plaine, barrée à l'aval par des cordons sableux, favorise alors le stockage des eaux de pluie et ne permet pas l'évacuation en direction de la mer.

\section{3 - Hydrographie, hydrologie}

Située à $5 \mathrm{~km}$ à l'amont des 2 barrages (Mohammed V et Hamadi), la station hydrologique de Melg El Widane, enregistre l'apport liquide total du bassin-versant. L'apport moyen annuel de la Moulouya est de 768 millions de $\mathrm{m}^{3}$ d'eau.

Les débits enregistrés à cette station ont un caractère parfois torrentiel. Ils sont formés dans le bassin moyen et supérieur ou sur les principaux contributeurs (oueds Za, Melloulou, fig. 3). En été, le cours de la Moulouya peut être à sec et son alimentation se fait à l'approche du barrage Mohammed V par l'oued Za. La crue de 1963 a détruit ainsi les premiers travaux du futur barrage Mohammed V, re-construit en 1967. Une autre crue importante a eu lieu en avril 1974 (3 $\left.300 \mathrm{~m}^{3} / \mathrm{s}\right)$. En novembre 1993, suite à des perturbations violentes sur le bassin amont, le débit a atteint $6429 \mathrm{~m}^{3} / \mathrm{s}$, obligeant les services du barrage Mohammed $\mathrm{V}$ à procéder à des lâchers vers le barrage Mechra Hamadi situé à l'aval. En raison des années de sécheresse précédentes, ces derniers lâchers ont été peu importants par rapport aux apports d'amont: les barrages jouent ainsi un rôle écrêteur de crue grâce à l'espace disponible de leurs bassins (fig. 4).

Les données hydrologiques disponibles (1961-1995) (SNOUSSI ET IMASSI, 2003) ont été utilisées pour calculer 


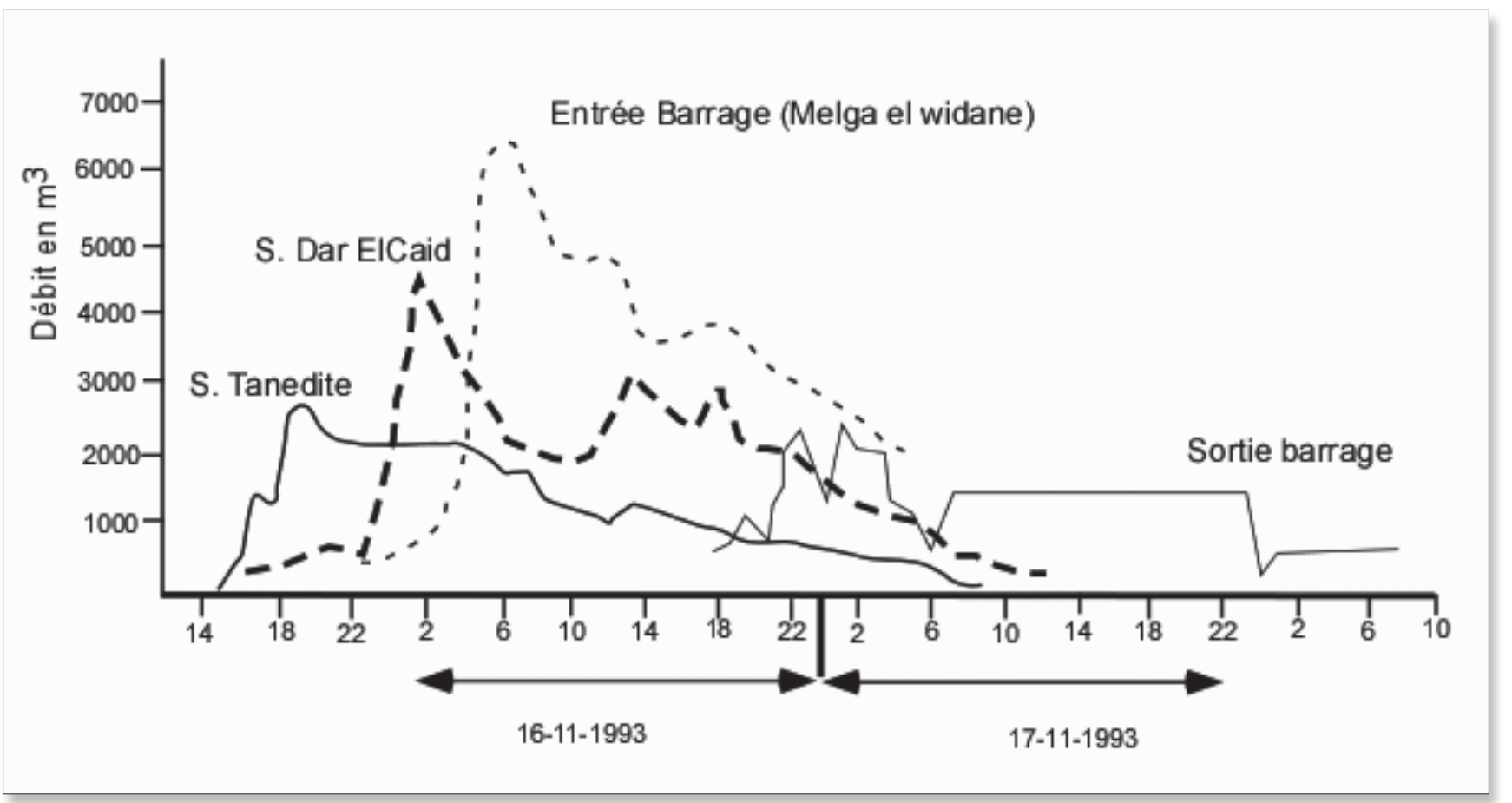

Fig. 4 - Débit de la Moulouya lors de la crue du 16 novembre 1993 aux stations situées à l'amont de Melga el Widane principale confluence des affluents de la Moulouya avant le barrage Mohammed V - Débit d'entrée enregistré à Melga el Widane et débit de sortie au barrage - D'après Messaoudi et Chigri 1994, modifié

le coefficient hydroclimatique (EC), qui permet de décrire les variations interannuelles : EC \% $=[\mathrm{Qma}-\mathrm{Qmi}] / \mathrm{Qma}$ où Qma est la moyenne des écoulements annuels et Qmi le total des écoulements interannuel moyens.

La variation de ce coefficient depuis 1960 (fig. 5) montre une baisse sensible de 1967 à 1990, qui reflète la sécheresse que le Maroc a subi pendant cette période (STOCKTON, 1993 in SNOUSSI et IMASSI, 2003). Entre 1990 et 1994, l'impact de la sécheresse est moins grave dans le bassin de l'oued Moulouya par rapport à d'autres bassins du pays.

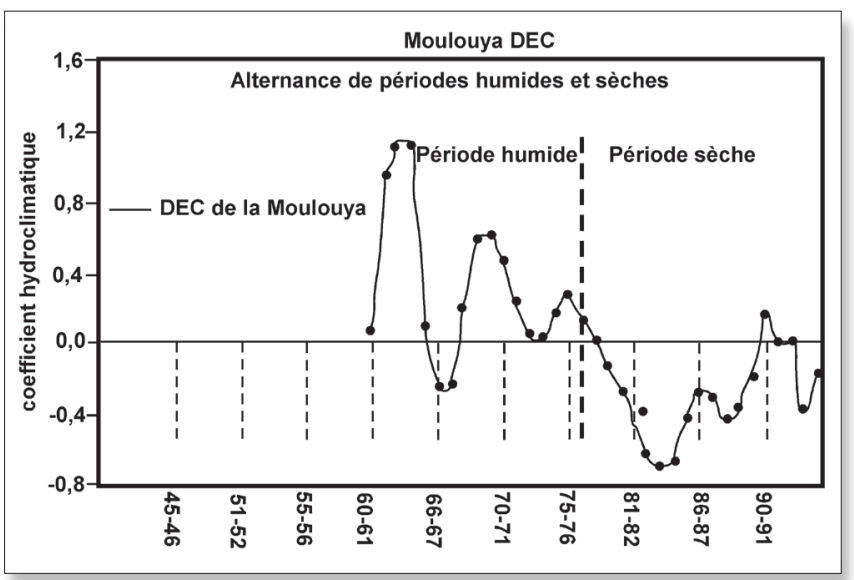

Fig. 5 - Coefficient hydroclimatique de la Moulouya - Snoussi et Imassi, 2003

\section{4 - Apports sédimentaires au littoral}

Étant données la nature du substrat dominé en particulier par les roches marneuses, qui constituent la moyenne Moulouya, la faible densité du couvert végétal et l'intensité des précipitations à l'amont, le barrage Mohammed V reçoit une eau très chargée en matières en suspension (MES).
Les concentrations de limons en suspension, mesurées aux 3 stations situées en amont proche du barrage Mohammed V, varient de 0,1 à $1,2 \mathrm{~kg} / \mathrm{m}^{3}$ pour les débits de base et les crues moyennes, et de 50 à $100 \mathrm{~kg} / \mathrm{m}^{3}$ lors des crues exceptionnelles (comme celles de 1963). En considérant une densité de 1,8 pour les matériaux constituant le bassin versant, la dégradation moyenne annuelle est donc de $156 \mathrm{t} / \mathrm{km}^{2} / \mathrm{an}$, correspondant à une érosion de $0,087 \mathrm{~mm} / \mathrm{an}^{2}$ (LALHOU A., 1984). Le volume initial du bassin du barrage Mohammed V (726 millions de $\mathrm{m}^{3}$ ) a déjà perdu pratiquement la moitié de son potentiel et risque d'être comblé en 2030, soit soixante ans après sa création.

Avant la construction des barrages toute la charge sédimentaire aboutissait à l'aval dans la plaine côtière. La plaine actuelle provient des alluvions déposées par la Moulouya lors des crues. Au cours de l'Holocène ces dernières ont permis la construction des terrasses limoneuses qui encadrent le lit actuel du fleuve. La figure 6 montre que la Moulouya a construit un lobe deltaïque encore progradant entre 1942 et 1958. Les cordons littoraux successifs, bien visibles sur les photos aériennes, décrivent l'avancée du trait de côte. Ils ont opposé une résistance à l'écoulement fluvial, favorisant le développement de méandres, puis leur recoupement. Le vaste cordon dunaire induit le développement de zones humides.

La crue de 1963 fait apparaître une nouvelle embouchure à l'ouest de la précédente par rupture du cordon dunaire et défluviation (fig. 7). L'importante inondation de la plaine littorale a alors été causée par la remontée de la nappe et l'accumulation des eaux en provenance des versants des Ouled Mansour. En 1980, une accumulation sableuse marque la nouvelle avancée de l'embouchure sur cette position récente, alors que les anciens bras forment des zones humides en cours de colmatage (fig. 8).

2 (NSTS) Nouvelle Station Touristique de Saïdia. 


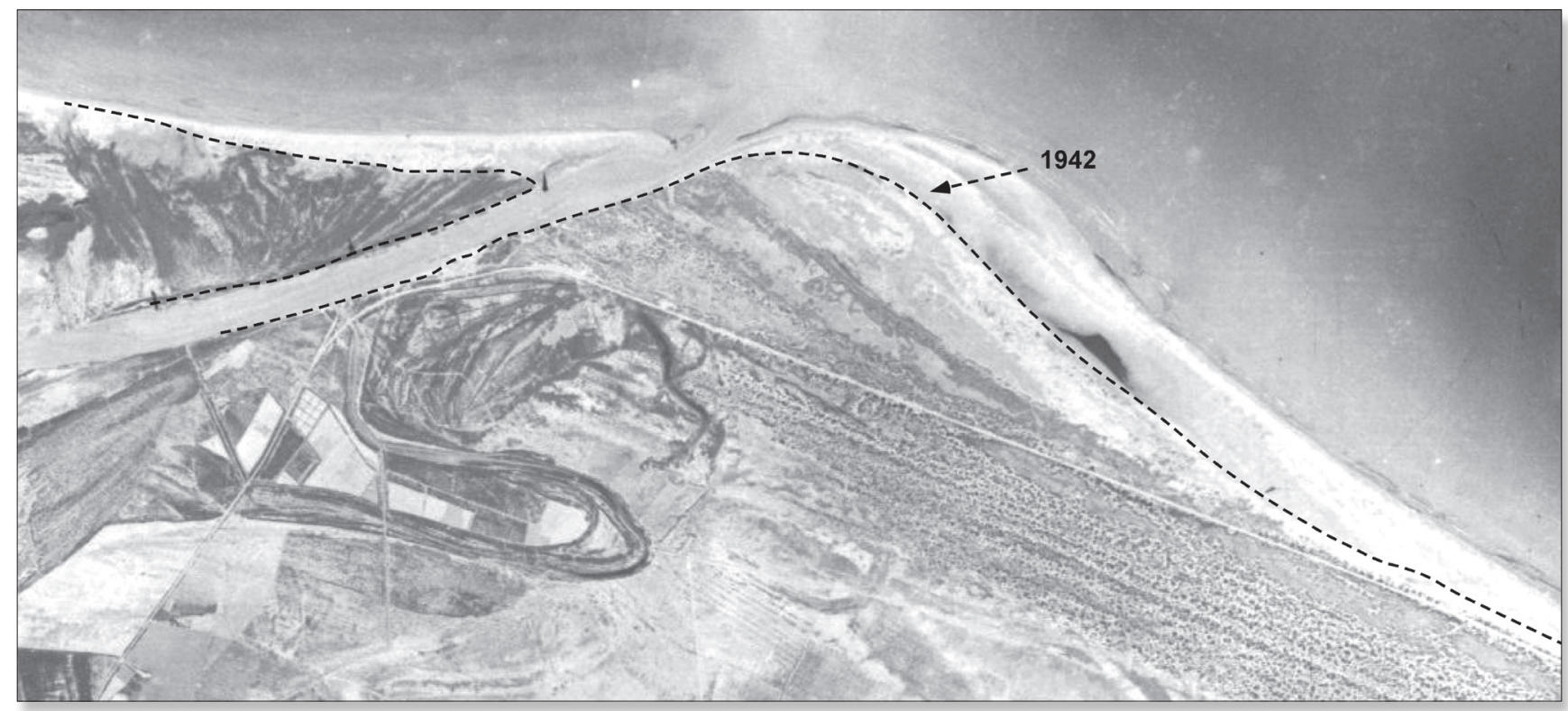

Fig. 6 - La plaine et le littoral de Saidia entre 1942 et 1958 - Photographie aérienne de 1958 de la Division de Cartographie (Rabat), modifiée d'après SNOuSSI et IMASSI 2003 pour le trait de côte en 1942

\section{5 - Dynamiques météo-marines}

On ne dispose d'aucune donnée concernant la houle, la fréquence des vagues et la vitesse des vents sur le littoral de Saïdia. Lors de l'étude de mise en place du port de plaisance de Saïdia, les données mises à disposition par le bureau d'étude responsable de l'étude technique provenaient de deux ports situés à une centaine de $\mathrm{km}$ de Saïdia!

La côte littorale de Maroc oriental ne subit que rarement des houles brutales. En hiver, comme souvent en Méditerranée, les houles deviennent plus importantes, pouvant atteindre $2 \mathrm{~m}$ de haut, associées à des vents en rafales qui peuvent accélérer la vitesse des vagues au littoral.
Nos observations de l'évolution de la plage montrent que la dérive littorale dominante est de direction $\mathrm{N}-\mathrm{O}$ à E-S-E. La déformation vers l'Est du tracé de l'embouchure de la Moulouya (fig. 8) et le remaniement de ses apports alluviaux vers la plage de Saïdia confirment ce constat.

L'apport alluvial de la Moulouya d'une part et l'étalement des sables par les vagues ont contribué à l'édification de la plage de Saida: les cordons parallèles de l'arrière côte attestent de la persistance de ces dynamiques depuis longtemps.

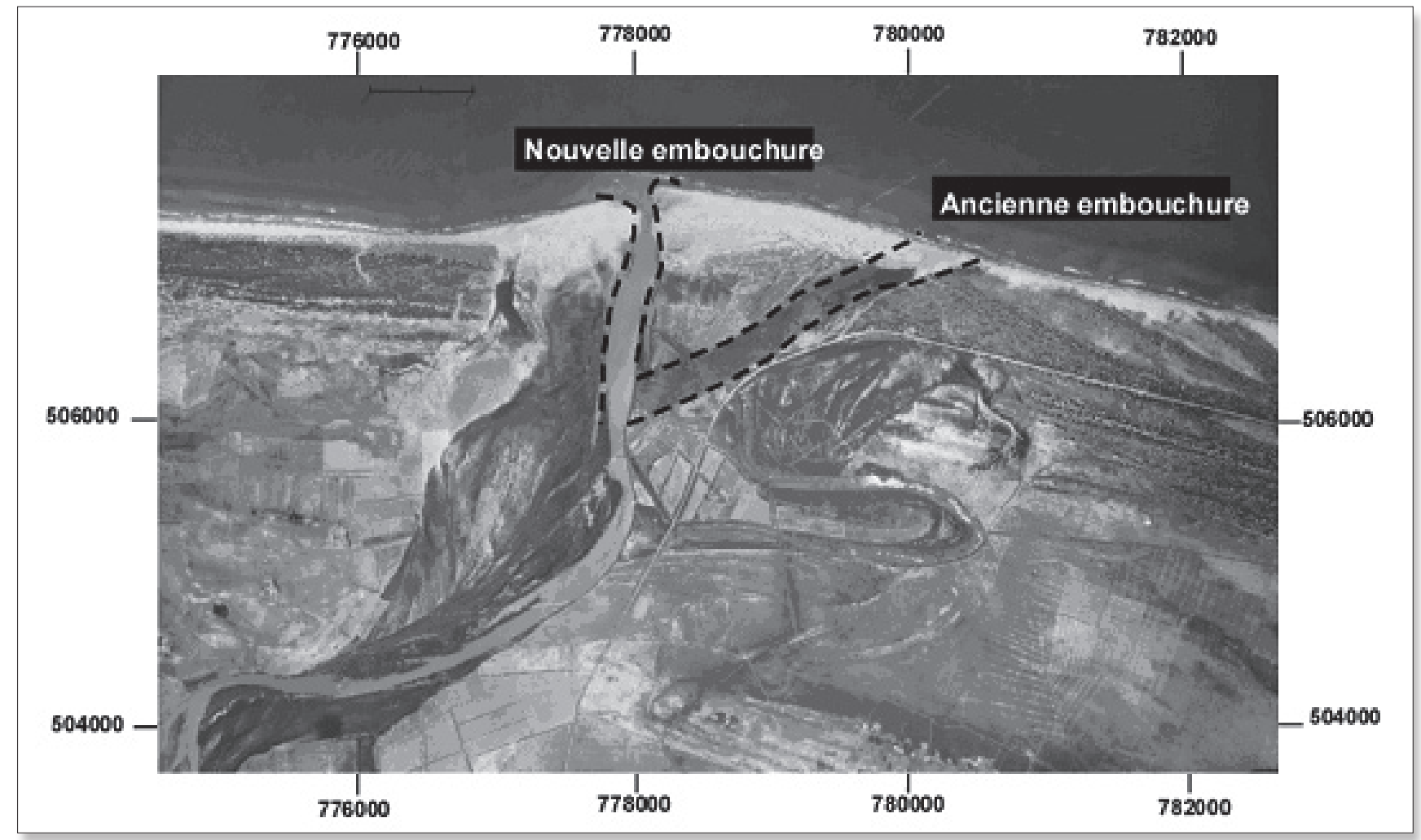

Fig. 7 - Une nouvelle embouchure apparaît à l'ouest de la précédente, issue de la rupture du cordon dunaire lors des fortes crues de 1963 Photographie aérienne de 1980 


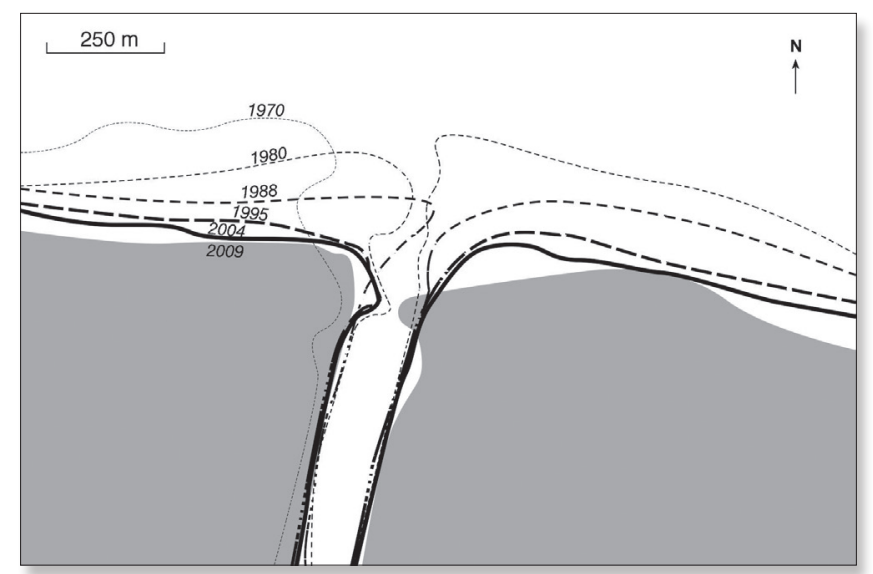

Fig. 8 - Formes et évolution de la nouvelle embouchure de la Moulouya entre 1970 et 2009

\section{3 - Impacts de la nouvelle station touristique de Saïdia (NSTS) et des aménagements de la zone limitrophe}

Le projet Saïdia-Mediterrania (fig. 9), d'une superficie de 713 ha, est situé à $60 \mathrm{~km}$ au nord d'Oujda et à quelques kilomètres à l'ouest de Saïdia. Initié par le promoteur immobilier espagnol FADESA, c'est le plus grand chantier touristique jamais réalisé au Maroc. Il comporte une marina (port de plaisance, centre commercial, base nautique,..), des hôtels de haut standing, des villas, des appartements, des résidences et des villages touristiques, 3 parcours de golf à 18 trous, un Aqua-park, un centre de thalassothérapie, un palais des congrès, une clinique et des équipements sportifs (piscine, stade de football, athlétisme, court de tennis).

Pour la mise à niveau de la région, d'importants travaux d'infra-structure ont été réalisés en matière de routes, d'adduction d'eau potable et de prélèvement d'eau pour l'irrigation des terrains de golf, d'électricité, d'assainissement, de protection contre les inondations.
Cet aménagement s'inscrit dans un environnement fragile, où la construction des barrages a réduit les apports sédimentaires au littoral. Le port de plaisance de Saïdia aggrave encore ce déséquilibre. La station touristique menace également les milieux naturels humides.

\section{I - Un littoral fragilisé par l’impact des barrages sur la Moulouya}

Avant la construction des barrages, l'évolution de trait de la côte était conditionnée essentiellement par les apports fluviatiles de la Moulouya lors de ses crues saisonnières, remaniés vers l'Est par la dérive littorale dominante, qui alimentait les plages de Saïdia.

À l'embouchure initiale, abandonnée lors de la crue de 1963 (fig. 7), le lobe deltaïque, encore présent en 1958, est déjà en recul à partir de 1970 et a complètement disparu en 2009: la côte a un tracé rectiligne, lissé par la dérive dominante d'Ouest.

Sur la nouvelle embouchure, apparue en 1963 (fig. 8), l'apport alluvial est réduit depuis la construction et la mise en service des barrages à partir de 1967. La déformation du lobe vers l'Est est visible dès 1980. Le recul du front deltaïque s'accélère ensuite : il atteint 7 à $8 \mathrm{~m} / \mathrm{an}$ entre 1980 et 2009. En 2009, le lobe d'embouchure de la Moulouya a disparu: la dérive littorale ne dispose donc plus de stocks sédimentaires susceptibles d'alimenter la plage de Saïdia.

\section{2 - Évolution du trait de côte du littoral de Saïdia de 1942 à 2009: impact du port de plaisance de Saïdia et de son extension sur le littoral}

Sur la plage de Saïdia, la dérive dominante d'Ouest a été interrompue par la construction du port de Saïdia en 1997, ce qui compromet gravement l'équilibre de la plage située à l'Est du port. Les mesures ${ }^{3}$ montrent déjà un recul rapide du littoral (en moyenne 8,1m/an entre 1958 et mai 2010).

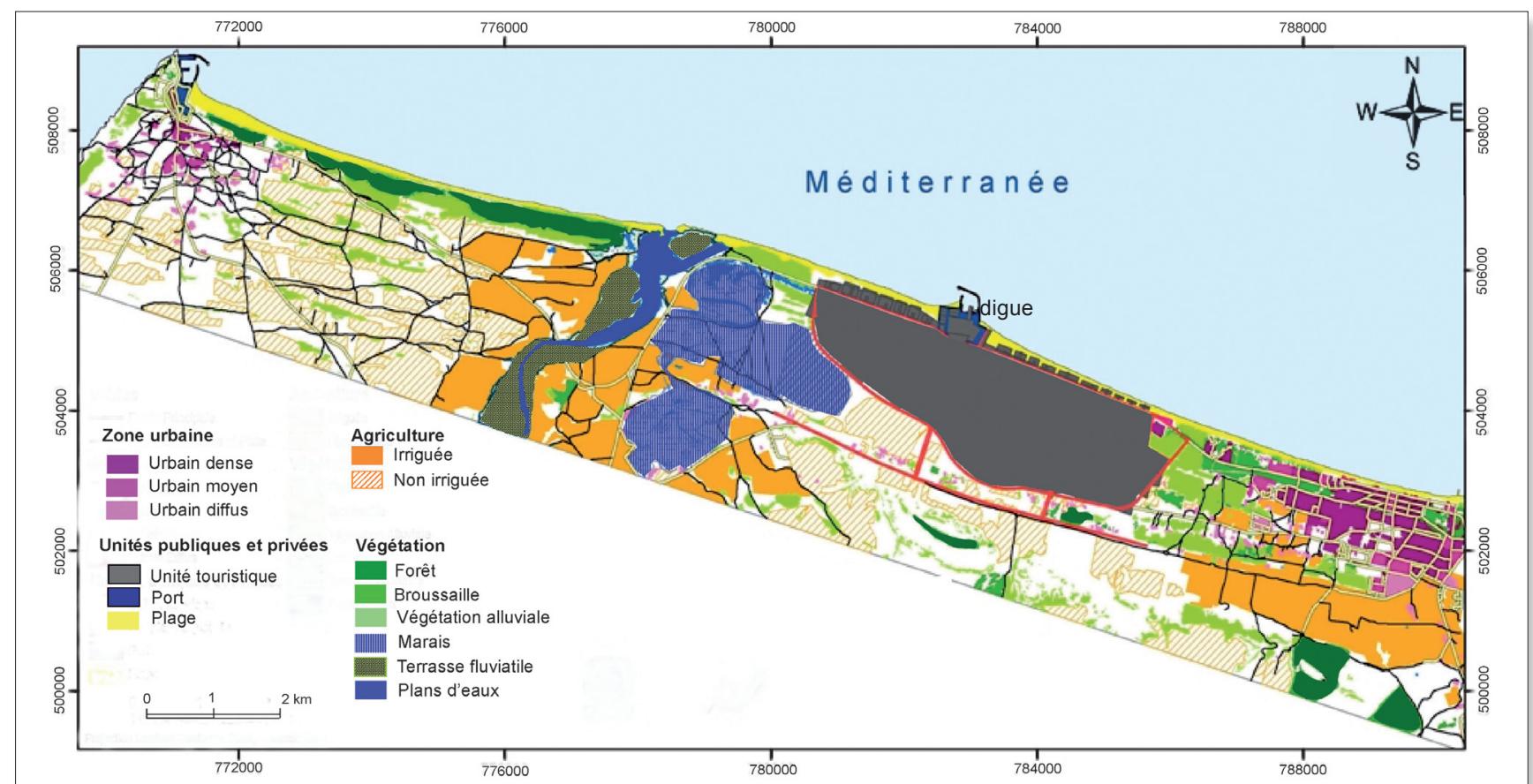

Fig. 9 - Carte d'occupation des sols de la zone littorale située entre Ras Kebdana et Saïdia, avec localisation de l'emplacement de la Station touristique de Saïdia-Fadesa - Snoussi et al., 2006

3 Les mesures avant 1996 (1958 à 1996) sont basées sur les photographies aériennes, tandis qu'à partir de 1996 jusqu'à 2010, se sont des mesures faites sur place et au même endroit chaque année au mois de février). 


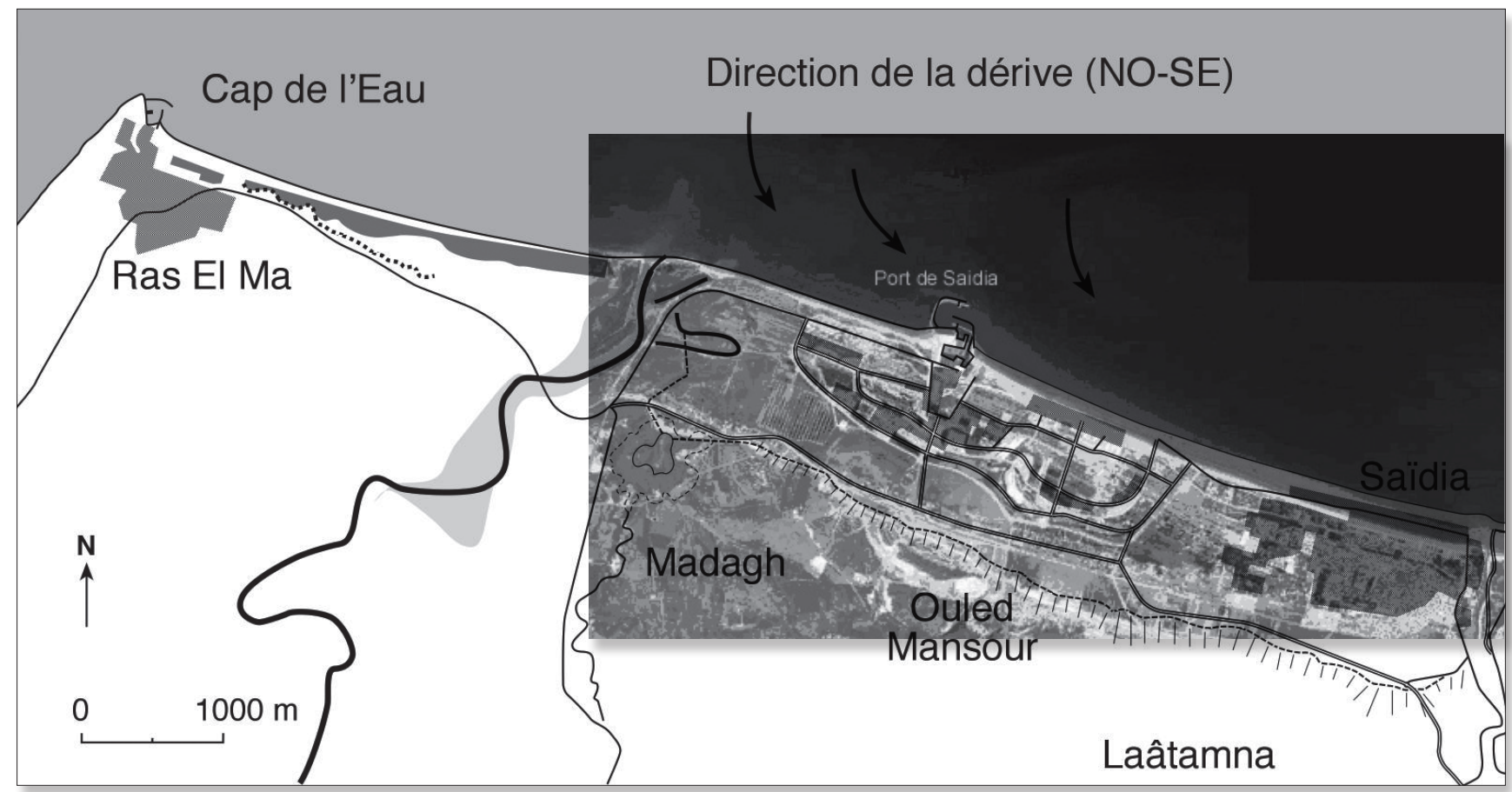

Fig. 10 - Nouvelle digue de la marina de plaisance du littoral de Saïdia montrant les perturbations des sédiments au voisinage du port - Cartographie: $P$. Pentsch d'après les indications de T. Boumeaza - Photomontage d'après P. Pentsch (cartographie) et Google (photographie aérienne)

La nouvelle digue de la marina de plaisance (fig. 9, 10), construite en 2008-2009 afin de stopper l'ensablement de l'entrée du port (nous n'avons pas de données chiffrées sur ce point) va encore accentuer les problèmes d'érosion de la plage. On peut observer sur l'image satellite (fig. 10) les perturbations du transit sédimentaire au voisinage du port, ainsi que la formation des barres d'avant côte et un début d'ensablement du côté Ouest du port.

Ainsi le transit sédimentaire qui se faisait en direction de l'Est et qui a contribué de façon permanente à la construction de la plage de Saïdia est actuellement stoppé.

D'autre part, une dérive littorale temporaire vers l'Ouest, pendant la période estivale, entraîne les sédiments en face de la nouvelle entrée du port, qui risque, peut être avec moins d'acuité, le même problème d'ensablement et de voir son entrée obstruée, nécessitant des opérations coûteuses de dragage. Donc à notre avis, et à l'encontre de ce que pensent plusieurs décideurs, le fait de changer l'orientation de l'entrée du port ne résoudra pas définitivement le problème d'ensablement auquel il a été confronté depuis sa construction: il faut prévoir un dragage en continu de son accès, malgré sa nouvelle digue.

\section{3 - Dégradations écologiques et géomorphologiques}

\subsection{I - Dégradation et anéantissement}

\section{du patrimoine naturel des dunes côtières}

Les cordons dunaires du littoral de Saïdia-Cap de l'eau sont des accumulations de sable, fixées ou mobiles, formant un système de dunes presque parallèles à la ligne de côte, qui matérialisent les anciennes positions du littoral. Leur chronologie a été étudiée par ZOURARAH (1995) et IRZI (2002). Elles ont un caractère environnemental et paysager important, puisqu'elles servaient de support à la forêt de Tazegraret, qui jouait un rôle écologique très important pour protéger l'arrière pays de l'ensablement en fixant les dunes. La forêt, qui comptait 550 ha à sa plantation en 1951, et encore en 1970, ne comprenait plus que 300 ha en 2004, au démarrage du projet de la NSTS.

Dans le cadre de la construction de la NSTS, le groupe FADESA Maroc s'est engagé, par la signature de la convention du 27 août 2003, à préserver et protéger l'environnement en général et spécialement la préservation des dunes, tel qu'il a été stipulé dans l'article 3-3 du cahier des charges urbanistiques approuvé en novembre 2003. De même, lors de la cession de cette forêt aux lotisseurs, les responsables des Eaux et Forêts souhaitaient que cet espace très fragile soit aménagé avec prudence tout en acceptant de légers aménagements.

En réalité le cordon dunaire a été entièrement décapé et les hôtels construits sur les dunes bordières en front de mer. Les constructions réalisées sur le haut de la plage et sur la dune bordière (promenade et Beach Clubs) ne peuvent qu'entraîner un amaigrissement puis un recul de la plage, du fait qu'elles entravent les échanges de sable entre la plage et les dunes et que ces dernières ne peuvent alors plus assurer leur rôle protecteur face à l'érosion marine et éolienne.

\subsection{2 - Impact des aménagements sur le SIBE de la Moulouya}

Les aménagements touristiques de la NSTS ont été effectués dans le SIBE de la Moulouya (fig. 1b) ou dans son voisinage immédiat sans tenir compte du statut particulier de ce site, de sa valeur écologique et son importance pour la biodiversité. Aucune étude d'impact, prévue par la loi marocaine, n'a été effectuée pour prévenir les préjudices et les dommages du SIBE. Les travaux effectués par les services de l'État, notamment par l'ONEP pour la réalisation de la STEP, par le Ministère des travaux publics pour la réalisation de la voie de contournement, par l'Agence du Bassin de la Moulouya pour la réalisation du canal d'évacuation des 
crues ou par la Direction des ports et du Domaine maritime pour la construction de la digue de la Marina de plaisance, risquent de causer des dommages irréversibles à la nature et à la biodiversité du SIBE par la fragmentation des habitats et la pollution. Ces infrastructures affectent en effet l'écoulement naturel de la nappe qui est la base de développement de cette zone humide. La construction du canal d'évacuation des eaux de surface contribue à une forte salinité de la nappe dans la partie qui borde directement la mer.

Nous pouvons considérer désormais que le SIBE de la Moulouya est une zone sinistrée, qui a été fortement dégradée à la suite des aménagements et la construction de la NSTS, réalisés dans le cadre de la nouvelle stratégie du Plan Azur.

\section{Conclusion}

Il ressort de cette étude que la construction de la NSTS, réalisée dans le cadre de la nouvelle politique du gouver- nement du Maroc pour développer le tourisme de masse, pose de graves problèmes environnementaux, qui menacent à terme les résultats attendus du Plan Azur lui-même. Ce constat rejoint celui qui a été établi sur la plupart des littoraux méditerranéens (PASKOFF, 1994).

Le port de plaisance, avec sa nouvelle digue, constitue maintenant une entrave aux apports sédimentaires de la Moulouya pour le bon maintien et l'équilibre de la plage. Son ensablement et du recul du trait de côte ne sont qu'un avant-goût des problèmes auxquels la ville de Saïdia et le complexe touristique Méditerrania-Saïdia risquent d'être confrontés dans les années à venir.

Les aménagements réalisés par FADESA à proximité de la plage annoncent une aggravation de la situation, avec l'amaigrissement puis le recul de la plage. Les dunes ne pourront plus assurer leur rôle protecteur face à l'érosion marine et éolienne. La probabilité de surélévation du niveau marin à l'avenir pourra aggraver la situation des infrastructures hôtelières qui bordent la mer.

\section{Références bibliographiques}

BoumeazA T., (1997), Morphologie et évolution du delta et du littoral de la basse Moulouya, Maroc nord oriental (Maroc): apport des images aéroportées et satellitaires. Géo observateur, nº 8, Rabat, p. 65-77.

- (2002), Le bassin inférieur de la Moulouya: Morphologie quaternaire, dynamique fluviale et morphologie côtière du littoral méditerranéen entre Kebdana et Saïdia, (Maroc oriental). Doctorat académique en Sciences, université de Liège, faculté des Sciences, mars 2002, 239 p.

- (2006 a), The impact of the dams on dynamics coast and the evolution of the Mediterranean beach of the North-East of Morocco, Workshop du CIESM, Monaco, 28 mars au 2 avril 2006, Fluxes of small and medium-size Mediterranean rivers: impact coastal areas. p. 75-83.

- (2006 b), Le littoral du Maroc nord-oriental (Maroc) : Environnement, impact des barrages et synthèse d'évolution de 1949 à 2005. Colloque en hommage à R. Paskoff, université d'Oujda, novembre 2006, p. 13-27.

Boumeaza T., SAIDI J., (2005), Infrastructures portuaires et risques environnementaux sur les littoraux: cas du littoral méditerranéen du Maroc oriental et du littoral atlantique de Mohammedia : étude par imageries aéroportées, colloque de l'Association des Géographes Marocains, en hommage à Hassan Benhalima, Publications de la faculté des Lettres et des Sciences humaines Fes-Saiss, p. 104-122

Hagelstein S., (2005), Gestion des risques naturels dans la plaine littorale de Saïdia (Maroc oriental), mémoire de $3^{\text {e }}$ cycle, université de Liège, FUSAGx, 45 p.

LAHLOU A., (1984), Envasement des barrages au Maroc, éditions Wallada, Rabat, 256 p.

IRZI Z., (2002), Les environnements du littoral méditerranéen du Maroc compris entre l'oued Kiss et le Cap des Trois Fourches. Dynamique sédimentaire et évolution et écologie des foraminifères benthiques de la lagune de Nador, thèse de doctorat, université Mohammed $1^{\text {er }}$, faculté des Sciences, Oujda, 311 p.

MAReK A., (2007), Analyse de la nouvelle station touristique de Saïdia (Maroc oriental) dans le cadre du développement durable, mémoire de licence, faculté des Sciences université de Liège, 156 p. + annexes.

Messouadi A., Chikri N. (1994), Crues de la Moulouya du 11 au 17/11/93, Eau et Développement, Rabat, n 17, juin 1994, p. 15-24.

NAHON C., (2008), Les apports de la télédétection dans l'étude diachronique de l'envasement de barrage: le cas des retenues Mohammed V et Hassan II (bassin de la Moulouya, Maroc oriental, mémoire de licence, faculté des Sciences, université de Liège, 156 p. + annexes

PASKOFF R., (1994), Les littoraux. Impacts des aménagements sur les littoraux, Masson, 256 p. 
Salmon M., Boumeaza T., SBAi A., Ben AtTA M., et Ozer A., (2010), L'érosion des côtes meubles de l'extrême nord-est du Maroc, Bulletin de la Société Géographique de Liège, nº 54, p. 97-106.

Sbai A, BoumeazA T., et Hagelsein S., (2005), Impact des actions anthropiques sur le littoral de Saïdia - Cap de l'Eau (Maroc Nord-Est). Colloque national "L'érosion anthropique au Maroc: méthodes diétude, extension et processus ", 23 et 24 décembre 2005, Kénitra, p. 161-182.

SBAi A., (2006), Impacts des projets d'aménagement sur le littoral de Saïdia-Cap de 1'Eau. Recueil des communications présentées lors de la journée d'étude La Protection de la Plage de Saïdia, organisée par l'Espace de Solidarité et de Coopération de l'Oriental le 08/07/2006, à paraître.

- (2008), L'érosion des côtes meubles du Maroc Nord-Est, $15^{e}$ rencontre des géomorphologues marocains: Le Maroc méditerranéen: Dynamique actuelle et mutations spatiales, université Abdelmalek Essaâdi, Tetouan, 29-30 mai 2008, à paraître.

SNOUSSI M., IMASSI M., (2003), Impact des fluctuations hydroclimatiques et des barrages sur l'évolution du trait de côte: delta de la Moulouya, université Mohammed V, faculté des Sciences, département de Géologie, Rabat, Maroc, inédit.

SnOussi M., OUCHANi T., NiAZI S., (2008), Vulnerability assessment of the impact of sea ]level rise and flooding on the Moroccan coast: the case of the Mediterranean eastern zone, Estuarine, Coastal and Shelf Science, 77, p. $206-213$.

ZouraraH B., (1995), La zone littorale de la Moulouya (Maroc nord-oriental): Transit sédimentaire, Évolution morphologique, Géochimie et état de la Pollution. thèse de $3^{\mathrm{e}}$ cycle, université Mohammed V, faculté des Sciences, département de Géologie, Rabat, Maroc, 197 p. 